Farid Zerimech, Merce Jourdain, Brigitte Onraed, Marion Bouchecareilh, Boualem Sendid, Alain Duhamel, Malika Balduyck and Pascal Pigny*, on behalf of the LICORNE Study Group ${ }^{\text {a }}$

\title{
Protease-antiprotease imbalance in patients with severe COVID-19
}

https://doi.org/10.1515/cclm-2021-0137

Received December 14, 2020; accepted February 20, 2021;

published online March 8, 2021

Keywords: alpha-1 antitrypsin; ARDS; COVID-19; elastase; matrix metalloprotease-12 (MMP-12).

To the Editor,

Patients infected with SARS-CoV-2 may develop either mild or suddenly severe symptoms of acute respiratory infection for yet unknown reasons. However, risk factors of severe Coronavirus Disease-19 (COVID-19) such as age, cardiomyopathy, diabetes, hypertension, obesity and male sex have been identified. In severe cases, the worsening of hypoxemia,

Malika Balduyck and Pascal Pigny share joint senior authorship.

${ }^{\mathrm{a} S e e}$ Appendix for details.

*Corresponding author: Prof. Pascal Pigny, PharmD, PhD, CHU Lille, Laboratoire de Biochimie " Hormonologie, Metabolisme, NutritionOncologie », 59037 Lille, France, Phone : +33 3204 44246,

E-mail: pascal.pigny@chru-lille.fr. https://orcid.org/0000-00033926-4487

Farid Zerimech, CHU Lille, Laboratoire de Biochimie « Hormonologie, Metabolisme, Nutrition-Oncologie ", Lille, France; Univ. Lille, ULR

4483 - IMPECS - IMPact de l'Environnement Chimique sur la Santé humaine, Lille, France

Merce Jourdain, CHU Lille, Univ-Lille, INSERM UMR 1190, Pôle de Réanimation, Lille, France

Brigitte Onraed, CHU Lille, Laboratoire de Biochimie, Biologie Prédictive, Lille, France

Marion Bouchecareilh, Univ. Bordeaux, CNRS, INSERM, BaRITOn, U1053, Bordeaux, France

Boualem Sendid, CHU Lille, Univ Lille, INSERM UMR 1285, Institut de Microbiologie, Lille, France

Alain Duhamel, Univ. Lille, CHU Lille, ULR 2694 - METRICS: Évaluation des Technologies de Santé et des Pratiques Médicales, Lille, France Malika Balduyck, CHU Lille, Laboratoire de Biochimie « Hormonologie, Metabolisme, Nutrition-Oncologie ", Lille, France; and Faculté de Pharmacie et EA 7364 RADEME, Laboratoire de Biochimie et Biologie Moléculaire, Université de Lille, Lille, France characteristic of acute respiratory distress syndrome (ARDS), requires an admission in an intensive care unit (ICU) for ventilatory support [1]. Mortality in these patients is high, around $20-35 \%$ depending on series [1]. Post-mortem histopathological analysis of lungs from patients who died from COVID-19 showed bilateral diffuse alveolar damage consistent with an ARDS [2]. Other associated features frequently reported are intra-alveolar fibrin deposition and a strong fibrotic reaction [3], protein-enriched interstitial edema and inflammatory infiltrates dominated by lymphocytes [3] or by pro-inflammatory macrophages [2]. In several animal models of SARS-CoV-2 infection histological analysis of the lungs also demonstrated a progressive infiltration of alveolar cavities by inflammatory cells mainly $\mathrm{T}$ lymphocytes, macrophages but also neutrophils [4]. Regarding pathogenesis, we hypothesize that the release by neutrophils and/or alveolar macrophages of stored proteases such as neutrophil elastase (NE), matrix metalloprotease-12 (MMP-12) or both, contributes to the diffuse alveolar damage. Indeed these proteases are able to degrade elastin fibers [5] which are core components of the interstitial matrix in the airways and fundamental for their viscoelastic properties. The aim of our study was to determine whether severe COVID-19 patients with different outcomes have different pattern of circulating proteases (NE, MMP-12) and of the major antiprotease alpha-1 antitrypsin (A1AT) during their stay in ICU.

Thirty-nine patients admitted into the ICU of CHU Lille for intubation and mechanical ventilation following COVID-19 between 18 March and 5 May 2020 were included. This study is nested within the LICORNE clinical and biological database which received an Ethics Committee approval (CPP Nord-Ouest 4, ECH20/09). SARS-CoV-2 infection was confirmed by RT-qPCR analysis of nasopharyngeal swabs. Patients were followed up longitudinally during the ICU stay. Through reviewing of the electronic medical records patients were classified into two groups: patients who died during $(\mathrm{n}=10)$ vs. patients alive at the end of ICU stay $(n=29)$. Initial blood samples for routine inflammatory markers testing (C-reactive protein [CRP], haptoglobin, transthyretin) were drawn at 
intubation (T1) and then periodically while in ICU. For the current study, we measured proteases and antiproteases in the remaining serum samples collected at five time points of the follow-up i.e. T1 (baseline), T2 (days 2-4), T3 (days 5-7), T4 (days 8-10) and T5 (days 11-18). The first death occurred at day nine. At the time of sample acquisition and processing, investigators were unaware of the patients' outcome. Broncho-alveolar lavage fluids (BALF) collected in 23 COVID-19 patients at any time of their ICU stay for ARDS were analysed for MMP-12, NE and total protein levels (one sample per patient). To eliminate any bias linked to difference in sampling time between alive and dead patients, statistical analysis was performed on BALF samples taken between day one and day 11 of the ICU stay $(\mathrm{n}=15)$. Among these 15 patients, 10 were new with no available matched serum samples.

NE and MMP-12 serum and BALF levels were measured by ELISA using commercial kits (Abcam, Cambridge, UK). A1AT serum level was measured by an immunoturbidimetric automated method [6]. The reference values ranged from 0.9 to $2 \mathrm{~g} / \mathrm{L}$ as commonly accepted [7]. Elastase inhibitory capacity (EIC) was determined by a kinetic spectrophotometric method based on the inhibition by A1AT of the hydrolytic activity of porcine pancreatic elastase on a chromogenic substrate as described [8]. The reference values of EIC determined in healthy subjects ranged from 17,500 to 31,500 inhibitory units (IU)/L [8].

A1AT immunophenotyping was performed by isoelectric focusing as described [9]. Total protein amount was measured by Pierce BCA kit. Categorical variables were compared by Fisher's exact test. Quantitative variables were compared by Mann Whitney U test. Linear mixed regression model (covariance pattern model) was used to evaluate the association of outcome with repeated biomarker measures before the first occurrence of death. Statistical analyses were performed with SPSS.

At the time of admission in the ICU (T1), COVID-19 patients with a positive outcome did not differ from patients who deceased in terms of age, Body Mass Index, sex-ratio, co-morbidities, or circulating levels of routine biochemical markers (Table 1). All patients displayed increased levels of acute phase proteins (CRP, haptoglobin, fibrinogen [Table 1]) testifying an intense inflammatory response. Serum A1AT, another acute phase protein, and, consequently, EIC were increased in both groups of patients, but weakly (median $\cong 1.5$ fold the upper normal value (UPN) Figure 1A). By contrast, MMP-12 and $\mathrm{NE}$ serum levels were strongly increased (median $\cong 10$ or 4-7 fold the UPN, respectively) in both groups with no significant differences between groups (Figure 1A). During the whole course of ICU stay, the peak value of MMP-12 was significantly higher in COVID-19 patients who will decease later on than in those with a positive outcome; a similar non-significant increase was observed for $\mathrm{NE}$ (Figure 1B). Peak value of EIC did not differ between the two groups. Analysis of the longitudinal evolution of NE, MMP-12, A1AT and EIC levels before first death by mixed model showed that only NE tended to be influenced by outcome ( $p$ for time interaction=0.075; Figure 1C). More precisely, NE levels peaked at $\mathrm{T} 2 \mathrm{in}$ patients who deceased ( $\mathrm{p}=0.001)$. The longitudinal evolution of the protease/ antiprotease ratios (NE/EIC and MMP-12/EIC) depicted in

Table 1: Baseline characteristics of COVID-19 patients.

\begin{tabular}{|c|c|c|c|c|c|}
\hline \multirow{2}{*}{$\begin{array}{l}\text { Clinical findings } \\
\text { Age, years }\end{array}$} & & \multirow{2}{*}{$\begin{array}{r}\text { Patients alive } \\
\mathrm{n}=29\end{array}$} & \multirow{2}{*}{$\begin{array}{r}\text { Patients deceased } \\
\mathbf{n}=10\end{array}$} & \multicolumn{2}{|c|}{ p-Value } \\
\hline & & & & 0.7097 & ns \\
\hline BMI & & $35.0[21.0-45.0]$ & $28.00[26.0-52.2]$ & 0.7513 & ns \\
\hline Gender & & $20 \mathrm{M} / 9 \mathrm{~F}$ & $8 \mathrm{M} / 2 \mathrm{~F}$ & 0.6927 & ns \\
\hline Hypertension & & $16(55.2 \%)$ & $3(30.0 \%)$ & 0.4470 & ns \\
\hline Obesity (BMI $\geq 30)$ & & $15(51.7 \%)$ & $4(40.0 \%)$ & 0.7164 & ns \\
\hline NIDDM & & $9(31.0 \%)$ & $2(20.0 \%)$ & 0.6927 & ns \\
\hline Cardiovascular disease & & $2(6.9 \%)$ & $1(10.0 \%)$ & 1.0000 & ns \\
\hline COPD & & $1(3.4 \%)$ & $1(10.0 \%)$ & 0.4521 & ns \\
\hline Dyslipidemia & & $2(6.9 \%)$ & $2(20.0 \%)$ & 0.2670 & ns \\
\hline Apnea & & $3(10.3 \%)$ & $3(30.0 \%)$ & 0.1628 & ns \\
\hline $\mathrm{CRP}, \mathrm{mg} / \mathrm{L}$ & $(<6.0)$ & 129 [38-383] & $101[28-330]$ & 0.0904 & ns \\
\hline Transthyrethin, g/L & $(0.2-0.4)$ & $0.1[0.04-0.41]$ & $0.08[0.03-0.18]$ & 0.4937 & ns \\
\hline Haptoglobin, g/L & $(0.3-2.0)$ & $4.14[2.91-8.78]$ & $4.04[0.77-6.72]$ & 0.1348 & ns \\
\hline Fibrinogen, $\mathrm{g} / \mathrm{L}$ & $(2.0-4.0)$ & $7.4[3.50-11.40]$ & $6.65[5.00-10.8]$ & 0.1519 & ns \\
\hline
\end{tabular}

COPD, chronic obstructive pulmonary disease; NIDDM, non-insulin-dependent diabetes mellitus; BMI, body mass index. Quantitative values are expressed as median [range]. For routine inflammatory markers, the usual values are indicated under brackets. 
Figure 1D is very similar to those of the corresponding protease i.e. NE or MMP-12 (Figure 1C). Indeed, the NE/EIC ratio also peaked at $\mathrm{T} 2$ in patients with a fatal outcome (Figure 1D). Moreover, A1AT immunophenotyping was conducted in 17 alive and eight deceased patients of our cohort. An unusual isoelectric focusing profile with anodic bands corresponding to highly sialylated MO and
M1 A1AT glycoforms [10] was observed in 100\% of patients with a negative outcome and in 59\% patients with a positive one (Figure 1E) ( $\mathrm{p}=0.057$, Fisher's exact test). Regarding BALF, samples taken from nine alive and six deceased patients were analyzed. Sampling time was not statistically different between the two groups (day median (alive vs. deceased): 3 vs. 4.5). The ratios NE/total protein

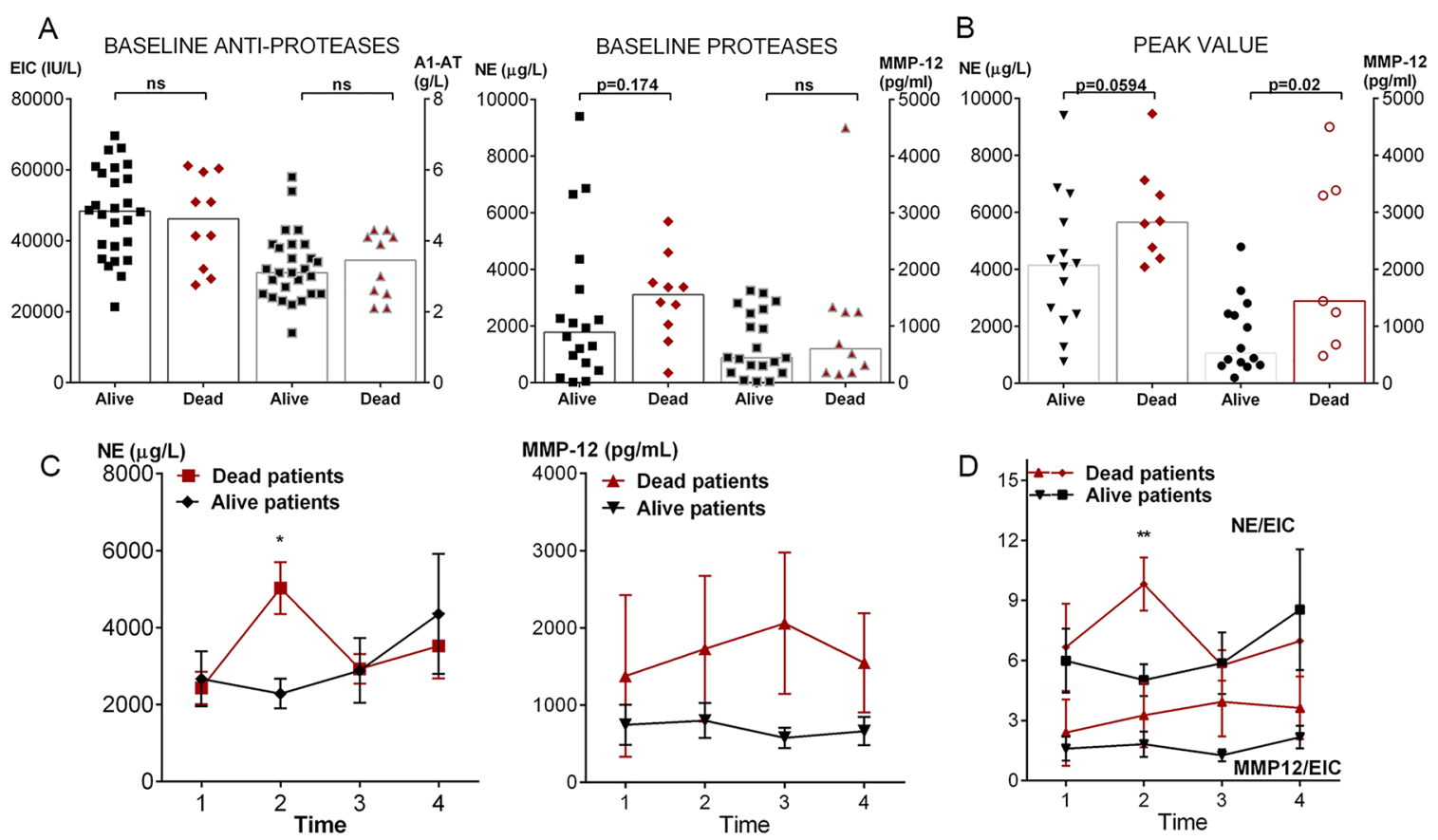

$\mathrm{E}$

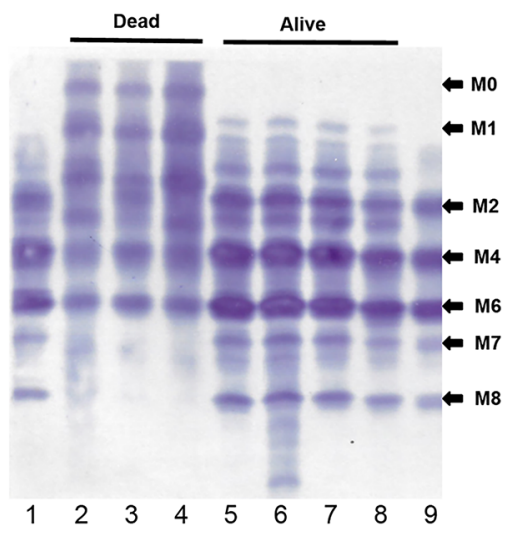

$\mathrm{F}$

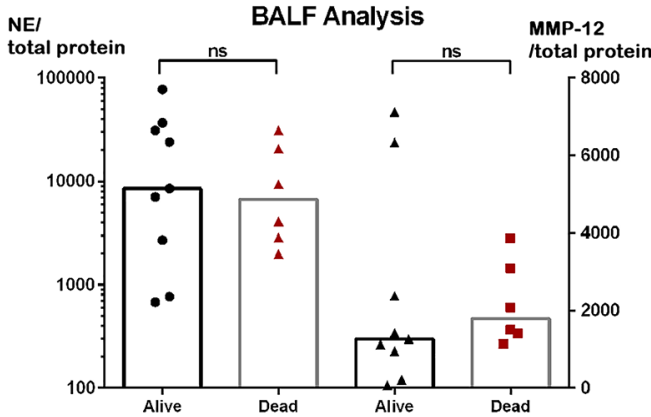

Figure 1: Proteases and antiproteases in severe COVID-19 patients.

(A) Serum levels of EIC (elastase inhibitory capacity), A1AT, NE and MMP-12 at admission into ICU (T1) of COVID-19 patients with ARDS according to their final outcome. The height of the box represents the median. ns, not significant. Normal range for A1AT: 0.9-2 g/L, for EIC: 17,50031,500 IU/L. (B) Peak value of NE and MMP-12 at the serum level during the whole ICU stay (T1-T5) according to patient's outcome. The height of the box represents the median. (C-D) Longitudinal follow-up of serum levels of NE, MMP-12, and protease/antiprotease ratios (NE/EIC or MMP12/EIC) in COVID-19 patients admitted into ICU before first death (median \pm SEM). The first death occurred at day 9 ( $n=1)$, the others at day $13(n=2)$ or later on $(n=7)$. The number of samples analyzed per subject during the follow up was: $2(1-4)$, median (range); ${ }^{\star} p<0.05$, ${ }^{\star \star} p<0.01$. (E) Analysis of serum A1AT phenotype by isoelectrofocusing demonstrated the presence of the anodic glycoforms M0 and M1 at different time points of the ICU stay for one representative patient who deceased vs. one representative patient with a positive outcome; 1, 9: Controls; 2: day nine; 3: day 11; 4: day 12; 5: day 0; 6: day one; 7: day two; 8: day three. (F) NE, MMP-12 and total protein concentrations were determined in 15 BALF samples collected in nine patients who survived and six patients who died during their ICU stay (one sample per patient taken between day one and day 11) and the NE or MMP-12/total protein ratios were calculated. ns, not significant. The height of the box represents the median. 
and MMP-12/total protein did not differ between deceased and alive patients (Figure $1 \mathrm{~F}$ ).

Our preliminary data showed that COVID-19 patients with fatal ARDS exhibited during their ICU stay significantly higher peak values of serum MMP-12 than those with a positive outcome. A similar trend was observed for NE. Moreover, NE levels evolution before first death tended to be associated with outcome with higher NE levels and NE/EIC ratio at T2 in deceased patients. This attests that a higher elastase activity is released from macrophages and neutrophils of COVID-19 patients with fatal ARDS. Concomitantly, they shifted their A1AT biosynthesis towards more anodic, more sialylated glycoforms in an attempt to boost its anti-inflammatory properties, as reported in patients with community-acquired pneumonia [10] or severe COVID-19 [11]. However, here the presence of the highly sialylated M0 and M1 glycoforms in COVID-19 patients is not correlated to the AAT serum levels or to the intensity of the inflammatory response (Sup Table 1), but to a higher NE/EIC ratio (Supplemental Table 1). Therefore, we postulate that the qualitative shift in A1AT glycoforms represents an attempt to trigger its anti-elastase activity. Unfortunately, this attempt was unsuccessful since the qualitative shift tends to be associated with a worse clinical outcome in COVID-19 patients.

Despite our cross-sectional analysis of BALF was negative, we postulate that an elastase-antielastase imbalance also exists locally during ICU stay leading to a net proteolytic activity in the lungs. Such an imbalance has been proposed as a pathogenic mechanism in emphysema [12]. Interestingly some COVID-19 patients developed pulmonary lesions complicated by a mediastinal emphysema as observed in patients with an A1AT genetic deficiency. Indirect arguments further support this similarity. In Italy, a geographical overlap between COVID-19 cases and A1AT deficient-patients was reported [13]. Obese male patients with a higher risk of severe COVID-19 had reduced serum A1AT levels and increased NE activity [14] suggestive of an elastase-antielastase imbalance. By contrast, female gender is positively correlated with serum A1AT levels [15]. In conclusion, this preliminary study is the first characterization of a protease-antiprotease imbalance in blood of COVID-19 patients with severe ARDS leading to death. We believe that our research could open new therapeutic perspectives in this setting with the introduction of pharmacological (as Sivelestat ${ }^{\circledR}$ or Alvelestat ${ }^{\circledR}$ ) or physiological inhibitors of NE such as A1AT prepared by plasma fractionation (Alfalastin ${ }^{\circledR}$, Prolastin ${ }^{\circledR}$ or Respreeza ${ }^{\circledR}$ ).
Acknowledgments: We thank Julien Labreuche for his help with statistical analysis, Catherine Lelorne, Jordan Soutif and Nadine François for their expert technical assistance, and Virginie Deprez for her help with protocol manadgment. The Lille COVID Research Network (LICORNE) wishes to acknowledge the contribution of residents, medical students, nursing teams, lab technicians, and clinical research associates in the midst of the SARS-CoV-2 pandemic.

Research funding: This work was funded by the Institut de Biochimie \& Biologie Moleculaire, CHU Lille (grant 8860). Author contributions: All authors have accepted responsibility for the entire content of this manuscript and approved its submission.

Competing interests: Authors state no conflict of interest. Informed consent: Informed consent was obtained from all individuals included in this study.

Ethical approval: This study is nested within the LICORNE clinical and biological database which received an Ethics Committee approval (CPP Nord-Ouest 4, ECH20/09).

\section{Appendix}

LICORNE Study Group Scientific Committee: Dominique Deplanque, Karine Faure, Guillaume Lefevre, Kazali Alidjinou, Régis Bordet, Ilka Engelmann, Delphine Garrigue, Anne Goffard, Eric Kipnis, Myriam Labalette, Marc Lambert, David Launay, Daniel Mathieu, Claude Alain Maurage, Julien Poissy, Martine Remy, Boualem Sendid, Sophie Susen, Maxime Caillier, Laurent Schwarb Laurent, Michael Hisbergues.

\section{References}

1. Bhatraju PK, Ghassemieh BJ, Nichols M, Kim R, Jerome KR, Nalla AK, et al. COVID-19 in critically ill patients in the Seattle region-case series. N Engl J Med 2020;382:2012-22.

2. Carsana L, Sonzogni A, Nasr A, Rossi RS, Pellegrinelli A, Zerbi P, et al. Pulmonary post-mortem findings in a series of COVID-19 cases form northern Italy: a two-centre descriptive study. Lancet Infect Dis 2020;20:1135-40.

3. Copin MC, Parmentier E, Duburcq T, Poissy J, Mathieu D, The Lille COVID-19 ICU and Anatomopathology Group. Time to consider histological pattern of lung injury to treat critically ill patients with COVID-19 infection. Intensive Care Med 2020;46:1124-6.

4. Bao LL, Deng W, Huang B, Gao H, Liu J, Ren L, et al. The pathogenicity of SARSCoV-1 in hACE2 transgenic mice. Nature 2020;583:830-3. 
5. Heinz A. Elastases and elastokines: elastin degradation and its significance in health and disease. Crit Rev Biochem Mol Biol 2020;55:252-73.

6. Balduyck M, Odou MF, Zerimech F, Porchet N, Lafitte JJ, Maitre B. Diagnosis of alpha- 1 antitrypsin deficiency: modalities, indications and diagnosis strategy. Rev Mal Resp 2014;31:729-45.

7. Dati F, Schumann G, Thomas L, Aguzzi F, Baudner S, Bienvenu J, et al. Consensus of a group of professional societies and diagnostic companies on guidelines for interim reference ranges for 14 proteins in serum based on the standardization against the IFCC/BCR/CAP Reference Material (CRM 470). International Federation of Clinical Chemistry. Community Bureau of Reference of the Commission of the European Communities. College of American Pathologists. Eur J Clin Chem Clin Biochem 1996;34:517-20.

8. Klumpp T, Bieth JG. Automated measurement of the elastaseinhibitory capacity of plasma with a centrifugal analyzer. Clin Chem 1979;25:269-72.

9. Zerimech F, Hennache G, Bellon F, Barouh G, Jacques Lafitte J, Porchet N, et al. Evaluation of a new Sebia isoelectrofocusing kit for alpha-1 antitrypsin phenotyping with the Hydrasis system. Clin Chem Lab Med 2008;46:260-3.

10. McCarthy C, Dunlea DM, Saldova R, Henry M, Meleady P, McElvaney OJ, et al. Glycosylation repurposes Alpha-1 antitrypsin for resolution of community-acquired pnemonia. Am J Respir Crit Care Med 2018;197:1346-9.

11. McElvaney OJ, McEvoy NL, McElvaney OF, Carroll TP, Murphy MP, Dunlea DM, et al. Characterization of the inflammatory response to severe COVID-19 Illness. Am J Respir Crit Care Med 2020;202: 812-21.

12. McGarry Houghton A. Matrix metalloproteinases in destructive lung diseases. Matrix Biol 2015;44-46:167-74.

13. Vianello A, Braccioni F. Geographical overlap between alpha-1 antitrypsin deficiency and COVID-19 infection in Italy: casual or causal ? Arch Bronconeumol 2020;56:609-10.

14. Mansuy-Aubert V, Zhou QL, Xie X, Gong Z, Huang JY, Khan AR, et al. Imbalance between neutrophil elastase and its inhibitor $\alpha 1$-antitrypsin in obesity alters insulin sensitivity, inflammation and energy expenditure. Cell Metabol 2013;17: 534-48.

15. Senn O, Russi EW, Schindler C, Imboden M, von Eckardstein A, Brandli O, et al. Circulating Alpha-1 antitrypsin in the general population: determinants and association with lung function. Resp Res 2008;9:35.

Supplementary Material: The online version of this article offers supplementary material (https://doi.org/10.1515/cclm-2021-0137). 